\title{
Effect of exercise and dietary intervention on serum metabolomics in men with insomnia symptoms: a 6-month randomized-controlled \\ trial
}

Xiaobo Zhang ${ }^{\text {a,b,c }}$, Xiuqiang Wang ${ }^{\text {b,c }}$, Shenglong Le ${ }^{b, c, d}$, Xiaowei Ojanen ${ }^{a, b}$, Xiao Tan ${ }^{\mathrm{e}}$, Petri Wiklund ${ }^{\mathrm{b}, \mathrm{c}}$, Sulin Cheng $*$ b,c,d.

${ }^{a}$ School of Life Sciences and Biotechnology, Shanghai Jiao Tong University, Shanghai, 200240, China

${ }^{b}$ Key Laboratory of Systems Biomedicine (Ministry of Education), and Exercise Translational Medicine Center, Shanghai Center for Systems Biomedicine, Shanghai Jiao Tong University, Shanghai, 200240, China

${ }^{c}$ Exercise, Health and Technology Center, Department of Physical Education, Shanghai Jiao Tong University, Shanghai, 200240, China

${ }^{d}$ Faculty of Sport and Health Sciences, University of Jyväskylä, Jyväskylä, 40014, Finland

${ }^{e}$ Department of Neuroscience, Uppsala University, Uppsala, 75123, Sweden

* Corresponding author:

E-mail address: shulin.cheng@jyu.fi (Sulin Cheng). 


\begin{abstract}
Background: Accumulating evidences have shown that lifestyle interventions such as exercise and diet are associated with improved sleep quality. However, the underlying molecular mechanisms remain unclear. Assessing exercise and diet intervention associated changes in circulating metabolomics profile in people with insomnia symptoms may help to identify molecular biomarkers that may link lifestyle changes to improved sleep outcomes.
\end{abstract}

Methods: The present study is a part of a 6-month randomized lifestyle intervention on sleep disorder subjects. Seventy-two Finnish men (aged: $51.6 \pm 10.1$ years; body mass index, BMI: $29.3 \pm 3.9 \mathrm{~kg} / \mathrm{m}^{2}$ ) with chronic insomnia symptoms who were assigned into different intervention groups completed this study (exercise $n=24$, diet $\mathrm{n}=27$ and control $\mathrm{n}=21$ ). The exercise group was assigned to a progressive aerobic exercise training with intensity of $60-75 \%$ of estimated maximum heart rate, $3-5$ times a week. The diet group aimed to reduce their total energy intakes by 300 to 500 kcal per day for the first three months. The control group were advised to maintain their current lifestyle. Sleep was assessed by using a non-contact sleep monitoring devise (Beddit sleep tracker). Blood samples were collected in the morning between 7:00 and 9:00 a.m. after overnight fasting. Gas Chromatography Time-Of-Flight Mass Spectrometry (GC-TOF-MS) method was used to determine the serum metabolites.

Results: Twenty-one metabolites were significantly changed in the exercise group, thirty-three metabolites in the diet group and five metabolites in the control group after intervention, respectively. The differential metabolites after exercise intervention were mainly related to glycerolipids and carbohydrates metabolism, while dietary intervention altered mainly amino acids metabolism and fatty acids metabolism related metabolites. We subsequently assessed the change of those metabolites with the change of sleep parameters and found that decreased alpha-ketoisocaproic acid ( $\mathrm{r}$ $=-0.52, p=0.026)$ was correlated with improved sleep efficiency (SE) in the exercise group. Change of 3-hydroxybutric acid $(r=-0.47, \mathrm{p}=0.025)$ and D-glucopyranose $(\mathrm{r}$ $=-0.54, \mathrm{p}=0.006)$ correlated negatively with SE in the diet group. On the other hand, oxalic acid $(\mathrm{r}=0.49, \mathrm{p}=0.021)$, D-glucopyranose $(\mathrm{r}=0.43, \mathrm{p}=0.048)$, 4-deoxyerythronic acid $(\mathrm{r}=0.60, \mathrm{p}=0.004)$ and tagatose $(\mathrm{r}=0.51, \mathrm{p}=0.016)$ correlated positively with change of SOL, and 2-keto-isovaleric acid $(r=0.45, p=$ 0.029) correlated with TST in the diet group. 
Conclusion: In conclusion, this study identified circulating metabolites that may represent a part of a biological mechanism through which lifestyle interventions are associated with improved sleep quality in people with insomnia.

Keywords: Diet, Exercise, Insomnia, Metabolomics 


\section{Introduction}

Insomnia, which is the most prevalent sleep disorder, is common in the modern society and have become an important public health issue ${ }^{1}$. The prevalence of insomnia symptoms was $21.4 \%$ in USA adults assessed by DSM-IV criteria ${ }^{2}$. Furthermore, sleep loss or sleep disruptions induced by insomnia symptoms are also associated with the increase in all-cause mortality and lead to detrimental effects on neuroendocrine systems ${ }^{1,3}$.

We have shown recently that six months exercise or diet intervention shortened objective sleep onset latency (SOL) and improved subjective sleep quality in overweight and obese men with insomnia symptoms, independent of weight loss ${ }^{4,5}$. Several human studies among insomniacs or individuals with other sleep disorders showed that exercise (including chronic and acute exercise) intervention have beneficial effects for sleep by increasing total sleep time (TST) and reducing wake after sleep onset (WASO) ${ }^{6-11}$. The acute aerobic exercise improved sleep quality by increasing activity of ascending brain serotonergic, while dietary supplementation of tryptophan (the only serotonin precursor) can reduce sleep onset latency ${ }^{12}$. The results of dietary intervention targeting patients with sleep disorders are conflicting ${ }^{13}$, with two studies showing isocaloric high fat intake associated with significantly better sleep than other diets in human ${ }^{4,14}$. A large cross-sectional study from China showed an association between decreased sleep duration and increased fat intake in 2828 adults ${ }^{15}$. However, the mechanisms underlying these associations between exercise/diet and sleep disorder, especially in insomnia remain largely are unclear.

Serum high-throughput metabolomics profiling is a feasible method to study the characteristic changes in small molecular metabolites in a pathophysiological state. Studies have shown that metabolites glucose, amino acid, fatty acid and tricarboxylic acid cycle intermediates levels differed in individuals with sleep disorders compared with healthy people ${ }^{16-18}$ as well as in animal studies ${ }^{19,20}$. However, no study has examined the effect of lifestyle intervention on circulating metabolome in patients with insomnia symptoms.Thus, we conducted the present study to assess the 
medRxiv preprint doi: https://doi.org/10.1101/2020.02.23.20026898; this version posted February 25, 2020. The copyright holder for this preprint

(which was not certified by peer review) is the author/funder, who has granted medRxiv a license to display the preprint in perpetuity.

All rights reserved. No reuse allowed without permission.

associations between serum metabolites and sleep quality after a randomized controlled six-month exercise or diet intervention in men with insomnia symptoms. 


\section{Methods}

\subsection{Subjects}

The study is a part of a 6-month randomized lifestyle intervention on middle-aged Finnish men with sleep disorders (including both insomnia and sleep apnea) ${ }^{21}$. For this report, we only included 72 men aged 30-65 years who self-reported having suffered from chronic insomnia lasting three months or longer and completed the 6-month intervention ${ }^{21}$. The subjects further filled a Vitalmed sleep questionnaire (which consists of all the questions in the Basic Nordic Sleep Questionnaire and the Epworth Sleepiness Scale (ESS)) were collected and reviewed by a physician. Of the qualified subjects and who completed the intervention, 24 subjects were allocated into exercise group, 27 subjects to diet group and 21 subjects to control group.

The study was approved by the Ethics Committee of the Central Finland Health Care District (7/2011 OTE) and registered under www.controlled-trials.com: ISRCTN77172005. Written informed consent was obtained from all participants before the baseline measurements and a copy of the signed consent form was archived.

\subsection{Background information and anthropometric measurements}

All lifestyle and medical history information were collected by self-administered questionnaires at the Laboratory of Sport and Health Sciences, University of Jyväskylä. Anthropometric measurements were performed after overnight fasting (12 h). Height was measured using a fixed wall scale device to the nearest $0.1 \mathrm{~cm}$. Weight was determined to the nearest $0.1 \mathrm{~kg}$ using an electronic scale and calibrated before each measurement session. BMI was calculated as weight $(\mathrm{kg})$ per height ${ }^{2}\left(\mathrm{~m}^{2}\right)$.

\subsection{Objective sleep measurement}

Seven-night sleep measurements both before and after intervention were performed for all participants by using a non-contact sleep monitoring system at their 
homes (Beddit sleep tracker, Beddit.com Oy, Espoo, Finland) ${ }^{22}$ and automatically analyzed by the Beddit server via internet. The system included a piezoelectric bed sensor. Ballistocardiographic signals were sampled by the piezoelectric sensor at 140 $\mathrm{Hz}$ and simultaneously uploaded to a web server through the Internet, where sleep/wake status was classified in 30-s epochs based on heart rate variability, respiration rate variability, and binary actigram. Total sleep time (TST), sleep onset latency (SOL, determined as the duration from being present in bed with lights-out to the first five minutes of consecutive sleep), wakefulness after sleep onset (WASO), and sleep efficiency (SE is commonly defined as the ratio of a person's total sleep time to time spent in bed, which refers to the amount of time a person is actually asleep during the time spent trying to sleep) were obtained of each night. Average values across the nights measured were used for analyses. The possible conditions which might affect the measurement, such as children and pets in the bedroom were recorded in the sleep diary. A research assistant visits each participant's home to set up the system before measurements started. The sleep data was validated by the piezoelectric system carried out against two-night polysomnography measurement (31 participants with insomnia complaints, aged $51.8 \pm 8.4$ years, $\mathrm{BMI}=30.9 \pm 4.8)^{5}$.

\subsection{Exercise intervention}

Exercise was prescribed according to the recommendations of the American College of Sports Medicine ${ }^{23}$. Nordic walking combined with other aerobic exercises was performed 30 to 60 minutes per session, 3 to 5 sessions per week, at the intensity level of $60-75 \%$ of estimated maximum heart rate. Two experienced exercise trainers were responsible for the instruction and supervision of exercise training once a week and in the other sessions, the participants exercised independently. In the beginning of each week, the trainer informed the participant of the duration and intensity (heart rate interval) of each exercise session ${ }^{5}$.

\subsection{Diet intervention}

Specific individualized diet program was developed after baseline assessments of 
each participant's current dietary intakes (based on three-day food diary) and body weight. The suggested diet contained the following relative macronutrient composition of energy intake: $40 \%$ carbohydrate with $<5 \%$ sucrose, $40 \%$ fat (saturated fatty acid (SAFA) 10\%, monounsaturated fatty acid (MUFA) $15-20 \%$, polyunsaturated fatty acid (PUFA) 10\%) and 20\% protein. Overweight/obese participants were advised to moderately reduce their total energy intake (by 300 to $500 \mathrm{kcal}$ per day for the first three months) with guidance on the proportion of macronutrients to be consumed. The target was to reduce body weight by $3 \mathrm{~kg}$ in the first three months of the intervention. Detail information was given in previous paper 4

\subsection{Control group}

Control group participants were instructed to maintain their habitual, pre-recruitment lifestyle during the intervention. They were invited to a lecture explaining the purpose of the group. After 6-month study period, they were given an opportunity to participate in the exercise plus dietary intervention program for 3 months.

\subsection{Serum sample collection}

Blood samples were collected in the morning between 7:00 and 9:00 a.m. after overnight fasting at baseline and after 6-month intervention from all subjects. Serum was extracted by centrifugation and stored immediately at $-80{ }^{\circ} \mathrm{C}$ until analyzed.

\subsection{Metabolomics assessment}

\subsubsection{Sample preparation}

Serum sample $(100 \mu \mathrm{L})$ was first thawed in room temperature, then mixed with methanol-chloroform (300 uL 3:1, v/v) solvent and L-2-chlorophenylalanine (10 $\mu \mathrm{L}$, $0.3 \mathrm{mg} / \mathrm{ml}$ in water) for metabolites extraction, and then kept at $-20^{\circ} \mathrm{C}$ for $10 \mathrm{~min}$. After centrifugation at $15000 \mathrm{~g}$ for $10 \mathrm{~min}, 300 \mu \mathrm{L}$ of supernatant was obtained and dried completely under nitrogen. Next, $80 \mu \mathrm{L}$ methoxyamine $(15 \mathrm{mg} / \mathrm{mL}$ in pyridine $)$ 
was added to the vial, vortexed for $30 \mathrm{~s}$ and kept at $37^{\circ} \mathrm{C}$ for $90 \mathrm{~min}$ followed by 80 $\mu \mathrm{L}$ BSTFA (1\% TMCS) and $20 \mu \mathrm{L} \mathrm{n}$-hexane at $70^{\circ} \mathrm{Cfor} 60 \mathrm{~min}$.

Quality control (QC) samples for serum were prepared by pooling equal aliquot of each sample and then were pretreated as serum samples, respectively.

\subsubsection{Gas Chromatography Time-Of-Filght Mass Spectrometry (GC-TOF-MS) analysis}

Each $1 \mu \mathrm{L}$ aliquot of the derivatized solution was injected in splitless mode into the Pegasus 4D GC-TOF-MS system (LECO Corporation, St Joseph, MI, USA). Separation was carried out on a non-polar DB-5 capillary column $(30 \mathrm{~m} \times 250 \mu \mathrm{m}$ I.D., J\&W Scientific, Folsom, CA), with high purity helium as the carrier gas at a constant flow rate of $1.0 \mathrm{~mL} / \mathrm{min}$. The $\mathrm{GC}$ temperature programming was set to begin at $90^{\circ} \mathrm{Cand}$ hold for $0.2 \mathrm{~min}$, and then followed by $10^{\circ} \mathrm{C} / \mathrm{min}$ oven temperature ramps to $180^{\circ} \mathrm{C}, 30^{\circ} \mathrm{C} / \mathrm{min}$ to $240^{\circ} \mathrm{C}, 20^{\circ} \mathrm{C} / \mathrm{min}$ to $280^{\circ} \mathrm{C}$, and a final 11 min maintenance at $280^{\circ} \mathrm{C}$. The temperature of injection and ion source was set to $280^{\circ} \mathrm{Cand} 220^{\circ} \mathrm{C}$, respectively. Electron impact ionization $(-70 \mathrm{eV})$ at full scan mode $(\mathrm{m} / \mathrm{z} 30-600)$ was used, Solvent delay: 7.6 minutes , with an acquisition rate of 100 spectrum/second in the MS setting.

\subsection{Data processing and statistics}

IBM SPSS statistics version 24 (SPSS, Inc., Chicago, IL, USA) was used to perform statistical analysis. All data were checked for normality by Shapiro-Wilk's W-test and for homogeneity by Levene's test before each analysis. Natural logarithm transformations were performed on non-normally distributed data. Spearman correlation analysis was performed with change percentage of metabolites and change percentage of parameters related to sleep. Between-group differences were evaluated by repeated measures ANOVA, followed with Sidak corrections for multiple comparisons. All tests were two sided with significance level $\mathrm{p}<0.05$. 
To account for heteroscedasticity data were normalized by log2-transformation. Multivariate statistical analysis and univariate analysis were used for metabolomics data analysis.

The acquired MS data from GC- MS were analyzed by ChromaTOF software (v 4.34, LECO, St Joseph, MI). The data sets resulting from GC-MS were separately imported into SIMCA-P+ 14.0 software package (Umetrics, Umeå, Sweden). Principle component analysis (PCA) and (orthogonal) partial least-squares-discriminant analysis (O) PLS-DA were carried out with SIMCA-P and differential metabolites were based on variable importance in the projection (VIP) > 1.0 and student $t$ test $\mathrm{p}<0.05$. In the present study, a 7-fold cross validation procedure and 200 random permutations tests were performed to avoid overfitting of the supervised OPLS-DA models. The intensity of each metabolite was used as final results. 


\section{Results}

Characteristics of the study participants are shown in Table 1. Briefly, during the 6-month intervention period, the control group had increased their body weight and fat mass (FM) significantly, while both intervention groups had no significant changes in body weight, FM, and total energy intake.

Of the sleep quality related variables, SOL decreased $(\mathrm{p}<0.05)$ after exercise and SE increased $(\mathrm{p}<0.05)$ after diet intervention. No significant changes in sleep-related parameters were found in the control group (Fig. 1).

A total of 210 known metabolites in serum among different groups were observed. The results show a clear separation between the baseline and follow-up (exercise $(\mathrm{R} 2 \mathrm{Y}=0.959, \mathrm{Q} 2=0.675)$, diet $(\mathrm{R} 2 \mathrm{Y}=0.969, \mathrm{Q} 2=0.823)$ and control $(\mathrm{R} 2 \mathrm{Y}=0.981, \mathrm{Q} 2=0.828))($ Supplementary Fig. S1). From Fig. S1B, 2D and 2F, the calculated R2 and Q2 values in permutation tests are lower than the original ones and the Q2 intercept on the vertical axis was less than zero. Therefore, the model is considered valid.

The significant metabolites in serum were screened according to the VIP value larger than 1 and $\mathrm{p}$ value $(\mathrm{p}<0.05)$ from the $t /$ test adjusted by false discovery rate (FDR). There were 21 metabolites affected by exercise intervention, 33 metabolites by diet intervention and 5 in the control group (VIP $>1$ and $\mathrm{p}<0.05$ ). All metabolites showed greater than 2-fold change between baseline and 6-months, except acetanilide in the exercise and control groups (Table S1). The significantly changed metabolites in all groups are shown in Fig. 2A, 2B and 2C. Figure 2D shows 2 unique metabolites altered in exercise group, 14 in diet group and 0 in control group.

We next assessed whether these metabolites were associated with changes in sleep parameters in each individual (Fig. 3). We found that decreased alpha-ketoisocaproic acid was correlated with improved SE in the exercise group $(\mathrm{r}=$ $-0.52, p=0.026)$. In addition, despite of no significant association between phosphate and SE, $67 \%$ of the participants showed increased phosphate levels after exercise intervention. In the diet group, change of 3-hydroxybutric acid $(r=-0.47, p=0.025)$ 
and D-glucopyranose $(\mathrm{r}=-0.54, \mathrm{p}=0.006)$ correlated negatively with SE. On the other hand, oxalic acid $(r=0.49, p=0.021)$, D-glucopyranose $(r=0.43, p=0.048)$, 4-deoxyerythronic acid $(\mathrm{r}=0.60, \mathrm{p}=0.004)$ and tagatose $(\mathrm{r}=0.51, \mathrm{p}=0.016)$ correlated positively with change of SOL, and 2-keto-isovaleric acid ( $\mathrm{r}=0.45, \mathrm{p}=$ 0.029) correlated with TST in the diet group. 


\section{Discussion}

In this study, the effects of exercise and dietary intervention on serum metabolome were assessed in men with insomnia symptoms. Twenty-one metabolites changed significantly after exercise intervention and thirty-three metabolites after dietary intervention. Five metabolites changed significantly in the control group during the same period.

Sleep efficiency (SE) is commonly defined as the ratio of a person's total sleep time to time spent in bed ${ }^{24}$. Early study have shown that sleep timing is associated with a large number of metabolites across a variety of biochemical pathways ${ }^{25}$. Widespread changes in circulating metabolites, including reduction of carbohydrates and increased levels of certain lipids and amino acids have been associated with sleep restriction and sleep deprivation ${ }^{16,17,26}$. However, such results may not reflect the alteration of circulating metabolites after exercise and dietary intervention in patients with insomnia symptoms. In this study, we found that concentrations of 21 metabolites changed significantly in subjects with insomnia symptom after exercise intervention. These metabolites were mainly related to glycerolipids metabolism and carbohydrates metabolism. We found that the decreased alpha-ketoisocaproic acid (KIC) was correlated with improved SE after exercise intervention. To our knowledge no previous study has linked exercise and KIC with sleep quality. KIC is metabolic intermediate in the metabolic pathway for $\mathrm{L}$-leucine ${ }^{27}$, which via leucine dehydrogenase and/or branched chain amino acid transferase at the expense of NH3, in a variety of tissues including skeletal muscle ${ }^{28}$. An early study by Funchal et al. ${ }^{29}$ showed that KIC increases phosphorylation of intermediate filament proteins from rat cerebral cortex by mechanisms involving $\mathrm{Ca} 2+$ and cAMP. Another study found that KIC and leucine provoke mitochondrial bioenergetic dysfunction in rat brain ${ }^{30}$. Thus, we speculate KIC and changes in the amino acid metabolism may play a role in exercise induced improvement in SE.

Phosphate was another metabolite associated with the change of sleep quality after 6-month exercise intervention. Phosphate is an essential mineral component of 
the human body involved on bone and cell membranes, molecules adenosine triphosphate, nicotinamide adenine dinucleotide, cyclic adenosine monophosphate and cyclic guanosine monophosphate metabolism ${ }^{31}$. Therefore, its dysregulation can affect the functionality of almost every organ system ${ }^{32}$. Phosphate balance is maintained by the kidneys and to some extent by the gastrointestinal tract in healthy adults, regulated by vitamin D, parathyroid hormone and phosphatonins such as fibroblast growth factor-23 (FGF23) ${ }^{31,33}$. Intrinsic factors including circadian rhythm, age and sex, and genetics, including single nucleotide polymorphisms (SNPs) are correlated with serum phosphate and mutations in the sodium-phosphate cotransporters and other hormone including dopamine, Angiotensin II and insulin like growth factor ${ }^{34}$. Patients with chronic kidney diseases showed that a higher sleep quality score was associated with higher phosphate levels ${ }^{35}$. Phosphate increased in our study after 6-month exercise intervention in patients with insomnia, consistent with previous study, which suggest appropriate concentration of phosphate is important for sleep ${ }^{35}$.

The effect of dietary intervention were more pronounced on sleep onset latency (SOL), which is the duration from being present in bed with lights-out to the first five minutes of consecutive sleep. We found that 33 metabolites were changed after dietary intervention. Particularly, increased oxalic acid, D-glucopyranose, 4-deoxyerythronic acid and tagatose are associated with improved SOL after dietary intervention. These metabolites are mainly related to purine metabolism, glucose metabolism, galactose metabolism.

Oxalic acid (oxalate) is derived from diet, degradation of ascorbate, and produced by the liver and erythrocytes. Oxalic acid has been shown to be reduced in both rat and human following sleep restriction, and recover to near baseline levels after sleep restriction ${ }^{36}$. In the present study, oxalic acid increased after 6-month diet intervention, supporting the notion that oxalic acid is a potential biomarker for sleep quality. In addition, 3-Hydroxybutyric acid (beta-hydroxybutyrate, 3-HB) which is an indicator of the status of hepatic fatty acid oxidation ${ }^{37,38}$, has been shown to increase in subcutaneous adipose after sleep loss in humans ${ }^{39}$. Serum 3-HB was also increased 
after diet intervention in patients with insomnia symptoms and was proposed to be related to the enhanced transport of plasma free fatty acid into the liver mitochondria 40. We also found increased D-glucopyranose after 6-month diet intervention. D-glucopyranose (glucose) is a monosaccharide containing six carbon atoms and an aldehyde group and is therefore referred to as an aldohexose which is a primary source of energy for living organisms. Abnormal glucose metabolism have been linked to disturbances of different aspects of sleep, including sleep duration, quality, respiratory function during sleep, and circadian timing ${ }^{41}$. Both short and long sleep durations are associated with worse glucose control in people with diabetes ${ }^{42}$. Recently, Kay.et al., showed that good sleepers, but not insomnia patients, had lower whole-brain glucose metabolism during recovery NREM sleep compared to baseline and individuals with insomnia. ${ }^{43}$.

In addition to oxalic acid, 3-HB and D-glucopyranose, we found increased tagatose and 2-keto-isovaleric acid after 6-month diet intervention. Tagatose (D-tagatose) has been associated with improved glucose control, weight loss and elevated high-density lipoprotein cholesterol ${ }^{44}$. In addition, 2-keto-isovaleric acid (KIV) arises from the incomplete breakdown of valine, which is one of branched-chain amino acids. KIV accumulate in Maple syrup urine disease (MSUD) patients, which is a metabolic disorder caused by a deficiency of the branched-chain alpha-keto acid dehydrogenase complex (BCKDC) ${ }^{45}$. However, no study has investigated the effect of tagatose and KIV on insomnia.

Our study has both strengths and limitations. The study participants were assigned to intervention and control groups at random, and sleep was measured using non-contact sleep monitoring system. Exercise training program was prescribed according to the recommendations of the American College of Sports Medicine and was supervised by experienced trainers. Diet intervention was individualized according the subjects body weight and caloric intake, and the duration of exercise and diet interventions were relatively long. The limitations include a small number of subjects and a relatively high drop-out rate in the control group, which may have affected the study results. 


\section{Conclusion}

In conclusion, exercise and diet intervention in patients with insomnia symptoms was associated improved sleep quality and changes in serum metabolites involved in glycerolipids metabolism, carbohydrates metabolism, purine metabolism, glucose metabolism. These results suggest circulating metabolites potentially involved in the exercise and diet induced improvement of sleep quality in patients with insomnia symptoms. 


\section{Figure legend}

Figure 1 The change of sleep parameters after intervention. TST means total sleep time, SOL means sleep onset latency, WASO means wakefulness after sleep onset, SE means sleep efficient.

Figure 2 The significant altered metabolites after intervention and venn figure of these metabolites in three groups. A means significant altered metabolites in exercise group, B means significant altered metabolites in diet group, C means significant altered metabolites in control group, and D showed common metabolites and separate metablites in three groups. Y-axis means standard data after $\log 2$ tranformation.

Figure 3 The intensity of significant changed metabolites in individuals between baseline and 6-month follow-up in exercise and diet groups. Each line represents one individual. 
Table 1 Characteristics of subjects (mean \pm SD)

\begin{tabular}{|c|c|c|c|c|c|c|c|c|}
\hline & \multicolumn{2}{|c|}{ Exercise $(n=24)$} & \multicolumn{2}{|c|}{ Diet $(n=27)$} & \multicolumn{2}{|c|}{ Control $(n=21)$} & \multicolumn{2}{|c|}{ Between-group } \\
\hline & Baseline & 6 months & Baseline & 6 months & Baseline & 6 months & 1 vs 3 & 2 vs 3 \\
\hline Age (years) & $51.2(9.7)$ & -- & $51.7(9.3)$ & -- & $50.7(9.3)$ & -- & & \\
\hline Weight (kg) & $92.3(14.6)$ & $92.5(13.9)$ & $93.8(11.8)$ & 92.7(11.9) & $93.1(17.2)^{\mathrm{c}}$ & $94.4(17.8)$ & 0.176 & 0.005 \\
\hline BMI & $29.3(4.0)$ & $29.4(3.8)$ & $29.4(3.7)$ & $29.0(3.7)$ & $29.2(4.4)$ & $29.4(4.6)$ & 0.612 & 0.047 \\
\hline $\begin{array}{c}\text { Total fat mass } \\
(\mathrm{kg})\end{array}$ & $27.3(9.5)$ & $27.2(9.0)$ & $27.4(8.4)$ & $26.8(8.6)$ & $28.0(9.7)^{\mathrm{c}}$ & $28.9(10.7)$ & 0.180 & 0.027 \\
\hline $\begin{array}{c}\text { Triglycerides } \\
(\mathrm{mmol} / \mathrm{L})\end{array}$ & $1.7(0.7)$ & $1.8(0.9)$ & $1.6(0.6)$ & $1.5(0.6)$ & $1.5(0.7)$ & $1.4(0.6)$ & 0.104 & 0.867 \\
\hline $\begin{array}{l}\text { Energy intake } \\
\quad(\text { kcal })\end{array}$ & $2285.2(561.6)$ & $2043.1(643.6)$ & $1981.2(372.1)$ & 1877.1(487.9) & $2223.5(410.1)^{\mathrm{c}}$ & $1920.9(463.7)$ & 0.588 & 0.606 \\
\hline VO2max & $29.3(9.3)^{\mathrm{a}}$ & $31.4(9.3)$ & $27.2(9.6)^{b}$ & $29.6(10.8)$ & $30.9(7.9)$ & $30.4(8.0)$ & 0.086 & 0.053 \\
\hline $\begin{array}{c}\text { Total energy } \\
\text { expenditure } \\
\text { (MET min/day) }\end{array}$ & $2349.9(224.8)$ & $2335.5(235.3)$ & $2346.1(215.9)$ & $2398.8(234.9)$ & $2341.1(242.8)$ & $2322.6(233.7)$ & 0.887 & 0.282 \\
\hline
\end{tabular}

a: exercise $0 \mathrm{~m}$ compare with exercise $6 \mathrm{~m}$; b: diet $0 \mathrm{~m}$ compare with diet $6 \mathrm{~m}$; c: control $0 \mathrm{~m}$ compare with control $6 \mathrm{~m}, \mathrm{P}<0.05$.

Between-group (1: exercise, 2: diet, 3: control) differences were evaluated by repeated measures ANOVA, followed with Sidak corrections for multiple comparisons. 
medRxiv preprint doi: https://doi.org/10.1101/2020.02.23.20026898; this version posted February 25, 2020. The copyright holder for this preprint (which was not certified by peer review) is the author/funder, who has granted medRxiv a license to display the preprint in perpetuity.

All rights reserved. No reuse allowed without permission.

Figure 1. The change of sleep parameters after intervention

TST

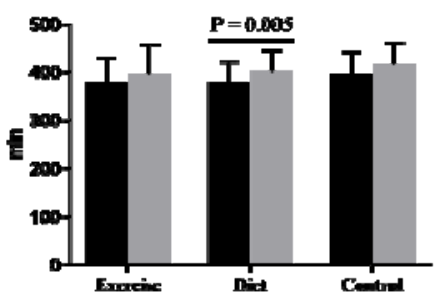

Waso

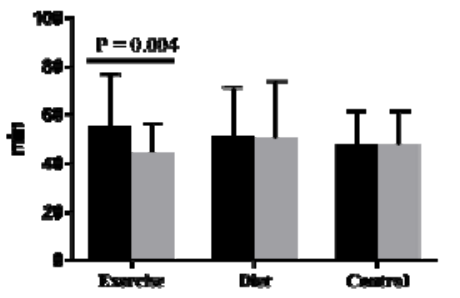

SOL

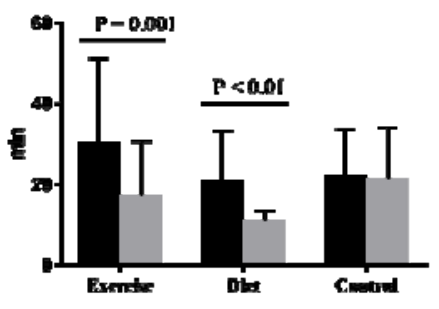

SE

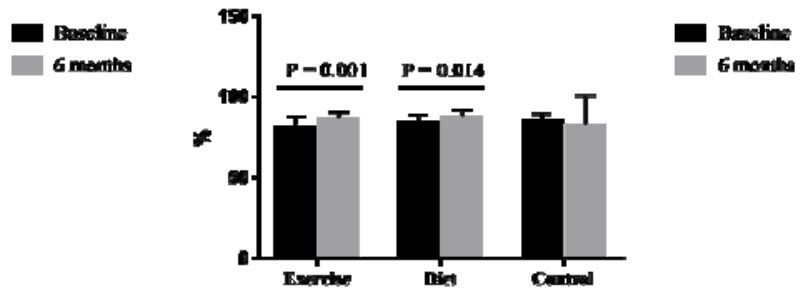


medRxiv preprint doi: https://doi.org/10.1101/2020.02.23.20026898; this version posted February 25, 2020. The copyright holder for this preprint (which was not certified by peer review) is the author/funder, who has granted medRxiv a license to display the preprint in perpetuity.

All rights reserved. No reuse allowed without permission.

Figure 2. The significant altered metabolites after intervention and venn figure of these metabolites in three groups
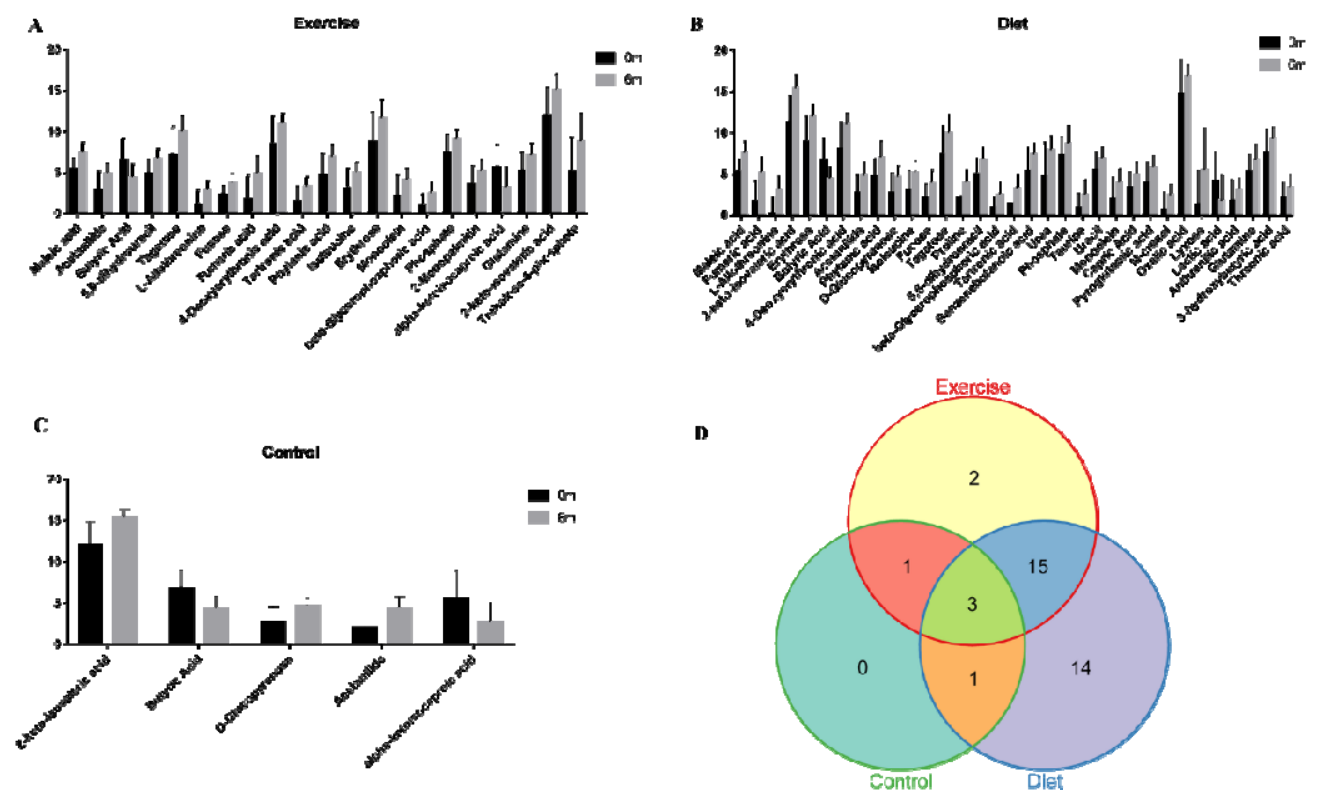
medRxiv preprint doi: https://doi.org/10.1101/2020.02.23.20026898; this version posted February 25, 2020. The copyright holder for this preprint (which was not certified by peer review) is the author/funder, who has granted medRxiv a license to display the preprint in perpetuity.

All rights reserved. No reuse allowed without permission.

Figure 3 The intensities of significant changed metabolites in exercise and diet group
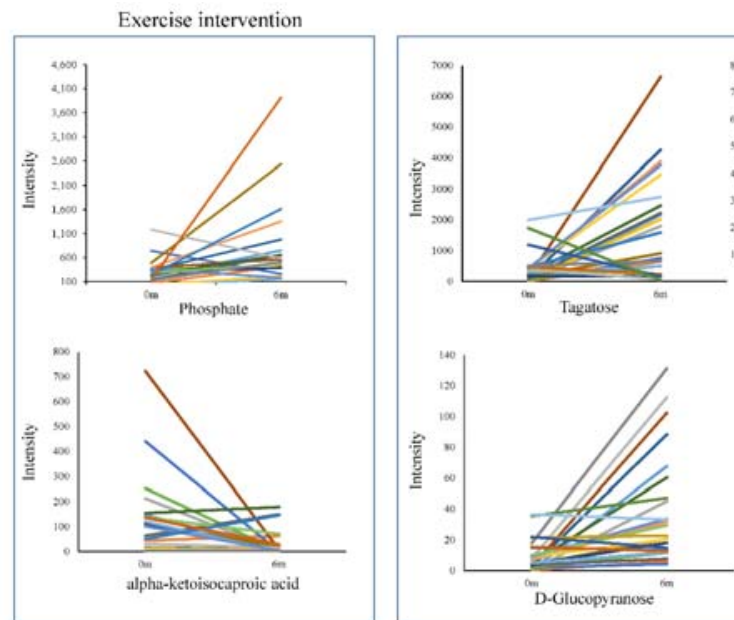

Dietary intervention

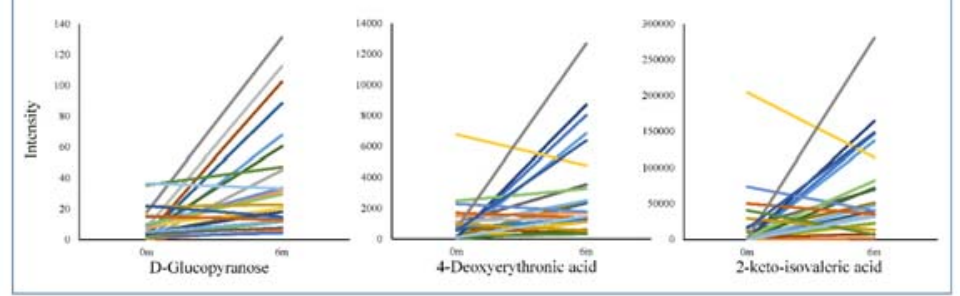




\section{Acknowledgments}

This study was supported financially by the by the Finnish Funding Agency for Technology and Innovation (TEKES2206/31/2010), the 111 Project (B17029) of Shanghai Jiao Tong University, the Shanghai Jiao Tong University Zhiyuan Foundation (Grant CP2014013), and China Postdoc Scholarship Council (201806230001).

\section{Authors' contributions}

The authors' contributions are as follows: SC is the principal investigator (PI). She designed the study and oversaw the implementation of the project, trained the researchers, supervised the doctoral students, and participated in the data collection, analyses and interpretation, and editing the manuscript. XZ, XW and PW drafted the manuscript. SL, XO and XT participated in the data analyses, interpretation and commenting the manuscript.

\section{Competing interests}

The authors declare that they have no competing interests. 


\section{References}

(1) Yazdi Z, K Sadeghniiat-Haghighi, Z Loukzadeh, K Elmizadeh, M Abbasi. Prevalence of Sleep Disorders and Their Impacts on Occupational Performance: A Comparison between Shift Workers and Nonshift Workers. Sleep Disord 2014;2014:870320.

(2) Budhiraja R, T Roth, DW Hudgel, P Budhiraja, CL Drake. Prevalence and polysomnographic correlates of insomnia comorbid with medical disorders. Sleep 2011;34(7):859-67.

(3) Cai XH, YP Xie, XC Li, WL Qu, T Li, HX Wang, et al. The prevalence and associated risk factors of sleep disorder-related symptoms in pregnant women in China. Sleep Breath 2013;17(3):951-6.

(4) Tan X, M Alen, K Wang, J Tenhunen, P Wiklund, M Partinen, et al. Effect of Six-Month Diet Intervention on Sleep among Overweight and Obese Men with Chronic Insomnia Symptoms: A Randomized Controlled Trial. Nutrients 2016;8(11).

(5) Tan X, M Alen, P Wiklund, M Partinen, S Cheng. Effects of aerobic exercise on home-based sleep among overweight and obese men with chronic insomnia symptoms: a randomized controlled trial. Sleep Med 2016;25:113-21.

(6) Passos GS, D Poyares, MG Santana, SA Garbuio, S Tufik, MT Mello. Effect of acute physical exercise on patients with chronic primary insomnia. J Clin Sleep Med 2010;6(3):270-5.

(7) Aiello KD, WG Caughey, B Nelluri, A Sharma, F Mookadam, M Mookadam. Effect of exercise training on sleep apnea: A systematic review and meta-analysis. Respir Med 2016;116:85-92.

(8) Driver HS, SR Taylor. Exercise and sleep. Sleep Med Rev 2000;4(4):387-402.

(9) Uchida S, K Shioda, Y Morita, C Kubota, M Ganeko, N Takeda. Exercise effects on sleep physiology. Front Neurol 2012;3:48.

(10) Kline CE, EP Crowley, GB Ewing, JB Burch, SN Blair, JL Durstine, et al. The effect of exercise training on obstructive sleep apnea and sleep quality: a randomized controlled trial. Sleep 2011;34(12):1631-40.

(11) Wiklund T, SJ Linton, P Alfoldi, B Gerdle. Is sleep disturbance in patients with chronic pain affected by physical exercise or ACT-based stress management? - A randomized controlled study. BMC Musculoskelet Disord 2018;19(1):111.

(12) Melancon MO, D Lorrain, IJ Dionne. Exercise and sleep in aging: emphasis on serotonin. Pathol Biol (Paris) 2014;62(5):276-83.

(13) Knowlden AP, CL Hackman, M Sharma. Systematic Review of Dietary Interventions Targeting Sleep Behavior. J Altern Complement Med 2016;22(5):349-62.

(14) Lindseth G, A Murray. Dietary Macronutrients and Sleep. West J Nurs Res 2016;38(8):938-58.

(15) Shi Z, M McEvoy, J Luu, J Attia. Dietary fat and sleep duration in Chinese men and women. Int J Obes (Lond) 2008;32(12):1835-40.

(16) Giskeodegard GF, SK Davies, VL Revell, H Keun, DJ Skene. Diurnal rhythms in the human urine metabolome during sleep and total sleep deprivation. Sci Rep 2015;5:14843.

(17) Bell LN, JM Kilkus, JN Booth, 3rd, LE Bromley, JG Imperial, PD Penev. Effects of sleep restriction on the human plasma metabolome. Physiol Behav 2013;122:25-31.

(18) Xu H, X Zheng, W Jia, S Yin. Chromatography/Mass Spectrometry-Based Biomarkers in the Field of Obstructive Sleep Apnea. Medicine (Baltimore) 2015;94(40):e1541.

(19) Zhan S, Y Wu, P Sun, H Lin, Y Zhu, X Han. Decrease in Circulating Fatty Acids Is Associated 
with Islet Dysfunction in Chronically Sleep-Restricted Rats. Int J Mol Sci 2016;17(12).

Sengupta A, SD Rhoades, EJ Kim, S Nayak, GR Grant, P Meerlo, et al. Sleep restriction induced energy, methylation and lipogenesis metabolic switches in rat liver. Int $\mathrm{J}$ Biochem Cell Biol 2017;93:129-35.

(21) Tan X, A Saarinen, TM Mikkola, J Tenhunen, S Martinmaki, A Rahikainen, et al. Effects of exercise and diet interventions on obesity-related sleep disorders in men: study protocol for a randomized controlled trial. Trials 2013;14:235.

(22) Paalasmaa J, M Waris, H Toivonen, L Leppakorpi, M Partinen. Unobtrusive online monitoring of sleep at home. Conf Proc IEEE Eng Med Biol Soc 2012;2012:3784-8.

(23) Garber CE, B Blissmer, MR Deschenes, BA Franklin, MJ Lamonte, IM Lee, et al. American College of Sports Medicine position stand. Quantity and quality of exercise for developing and maintaining cardiorespiratory, musculoskeletal, and neuromotor fitness in apparently healthy adults: guidance for prescribing exercise. Medicine and science in sports and exercise 2011;43(7):1334-59.

(24) Reed DL, WP Sacco. Measuring Sleep Efficiency: What Should the Denominator Be? J Clin Sleep Med 2016;12(2):263-6.

(25) Xiao Q, A Derkach, SC Moore, W Zheng, XO Shu, F Gu, et al. Habitual Sleep and human plasma metabolomics. Metabolomics 2017;13(5).

(26) Davies SK, JE Ang, VL Revell, B Holmes, A Mann, FP Robertson, et al. Effect of sleep deprivation on the human metabolome. Proc Natl Acad Sci U S A 2014;111(29):10761-6.

(27) Wilson JM, PJ Fitschen, B Campbell, GJ Wilson, N Zanchi, L Taylor, et al. International Society of Sports Nutrition Position Stand: beta-hydroxy-beta-methylbutyrate (HMB). J Int Soc Sports Nutr 2013;10(1):6.

(28) Matthews DE, R Harkin, A Battezzati, DJ Brillon. Splanchnic bed utilization of enteral alpha-ketoisocaproate in humans. Metabolism 1999;48(12):1555-63.

(29) Funchal C, A Zamoner, AQ dos Santos, SO Loureiro, M Wajner, R Pessoa-Pureur. Alpha-ketoisocaproic acid increases phosphorylation of intermediate filament proteins from rat cerebral cortex by mechanisms involving Ca2+ and cAMP. Neurochem Res 2005;30(9):1139-46.

(30) Amaral AU, G Leipnitz, CG Fernandes, B Seminotti, PF Schuck, M Wajner. Alpha-ketoisocaproic acid and leucine provoke mitochondrial bioenergetic dysfunction in rat brain. Brain Res 2010;1324:75-84.

(31) Manghat P, R Sodi, R Swaminathan. Phosphate homeostasis and disorders. Ann Clin Biochem 2014;51(Pt 6):631-56.

(32) Brown RB, MS Razzaque. Phosphate toxicity: a stealth biochemical stress factor? Med Mol Morphol 2016;49(1):1-4.

(33) Vervloet MG, S Sezer, ZA Massy, L Johansson, M Cozzolino, D Fouque, et al. The role of phosphate in kidney disease. Nat Rev Nephrol 2017;13(1):27-38.

(34) Lederer E. Regulation of serum phosphate. J Physiol 2014;592(18):3985-95.

(35) Zhang J, C Wang, W Gong, H Peng, Y Tang, CC Li, et al. Association between sleep quality and cardiovascular damage in pre-dialysis patients with chronic kidney disease. BMC Nephrol 2014;15:131.

(36) Weljie AM, P Meerlo, N Goel, A Sengupta, MS Kayser, T Abel, et al. Oxalic acid and diacylglycerol 36:3 are cross-species markers of sleep debt. Proc Natl Acad Sci U S A 
2015;112(8):2569-74.

(37) Zivin JA, JF Snarr. An automated colorimetric method for the measurement of 3-hydroxybutyrate concentration. Anal Biochem 1973;52(2):456-61.

(38) Brashear A, GA Cook. A spectrophotometric, enzymatic assay for D-3-hydroxybutyrate that is not dependent on hydrazine. Anal Biochem 1983;131(2):478-82.

(39) Cedernaes J, M Schonke, JO Westholm, J Mi, A Chibalin, S Voisin, et al. Acute sleep loss results in tissue-specific alterations in genome-wide DNA methylation state and metabolic fuel utilization in humans. Sci Adv 2018;4(8):eaar8590.

(40) Han P, Y Huang, Y Xie, W Yang, Y Wang, W Xiang, et al. Metabolic phenotyping in the mouse model of urinary tract infection shows that 3-hydroxybutyrate in plasma is associated with infection. PLoS One 2017;12(10):e0186497.

(41) Reutrakul S, E Van Cauter. Interactions between sleep, circadian function, and glucose metabolism: implications for risk and severity of diabetes. Ann N Y Acad Sci 2014;1311:151-73.

(42) Alnaji A, GR Law, EM Scott. The role of sleep duration in diabetes and glucose control. Proc Nutr Soc 2016;75(4):512-20.

(43) Kay DB, HT Karim, BP Hasler, JA James, A Germain, MH Hall, et al. Impact of acute sleep restriction on cerebral glucose metabolism during recovery non-rapid eye movement sleep among individuals with primary insomnia and good sleeper controls. Sleep Med 2019;55:81-91.

(44) Lu Y, GV Levin, TW Donner. Tagatose, a new antidiabetic and obesity control drug. Diabetes Obes Metab 2008;10(2):109-34.

(45) Sgaravatti AM, RB Rosa, PF Schuck, CA Ribeiro, CM Wannmacher, AT Wyse, et al. Inhibition of brain energy metabolism by the alpha-keto acids accumulating in maple syrup urine disease. Biochim Biophys Acta 2003;1639(3):232-8. 